\title{
Estrategias de aprendizaje para promover el cuidado medioambiental a través de la experimentación ${ }^{1}$
}

\section{Learning strategies to promote environmental care through experimentation}

DOI: http://dx.doi.org/10.17981/cultedusoc.9.3.2018.44

Artículo de investigación. Fecha de recepción: 15/06/2018. Fecha de aceptación: 27/11/2018

Felipe Ospino-Moya²;

Oladis Palomino-Sánchez; Martha Gómez-De Zambrano; Merly Infante-Moreno; Nubia Castro-De Utria; Norbelis Lascarro-Moya y Judith Saucedo-Yépez ${ }^{3}$

IED Bienvenido Rodríguez (Colombia) felipeson07@hotmail.com

Para citar este artículo:

Ospino-Moya, F., Palomino-Sánchez, O., Gómez-De Zambrano, M., Infante-Moreno, M., Castro-De Utria, N., Lascarro-Moya, N. y Saucedo-Yépez J. (2018). Estrategias de aprendizaje para promover el cuidado medioambiental a través de la experimentación. Cultura. Educación y Sociedad 9(3), 383-390. DOI: http://dx.doi.org/10.17981/cultedusoc.9.3.2018.44

\section{Resumen}

El desarrollo de la presente investigación busca apoyar el proceso de aprendizaje para la construcción de saberes y conocimientos sobre protección y conservación del medio ambiente, fomentando la educación ambiental con el uso adecuado de las relaciones con la naturaleza y de esta manera lograr el bienestar humano a través de prácticas que hagan posible una vida armónica con el entorno. Por tal razón el objetivo general es crear estrategias metodológicas para mejorar el proceso de aprendizaje. La investigación abordo una metodología cualitativa, de alcance descriptivo, con un diseño de Campo. Los participantes fueron los estudiantes de los grados $5^{\circ}, 6^{\circ}$ Y $9^{\circ}$ la Institución Educativa Bienvenido Rodríguez. Como conclusión se pude inferir que es la experimentación la que permite estrategias de enseñanzas novedosas y dinámicas que motivan a los estudiantes a participar y generar nuevos saberes a partir del descubrimiento.

Palabras clave: Aprendizaje; Estrategias metodológicas; experimentación; medio ambiente; bienestar humano.

\section{Abstract}

The development of this research seeks to support the learning process for the construction of knowledge and knowledge about protection and conservation of the environment, promoting environmental education with the appropriate use of relationships with nature and thus achieve human wellbeing. Through practices that make possible a harmonious life with the environment. For this reason, the general objective is to create methodological strategies to improve the learning process. The research addressed a qualitative methodology, of descriptive scope, with a Field design. The participants were the students of the 5th, 6th and 9th grades of the Bienvenido Rodríguez Educational Institution. As a conclusion, it could be inferred that it is experimentation that allows novel and dynamic teaching strategies that motivate students to participate and generate new knowledge based on discovery.

Keywords: Learning; Methodological strategies; experimentation; environment; human well-being.

1 Este artículo ha sido derivado del Proyecto Fortalecimiento de la Cultura Ciudadana y Democrática en CT+I a través de la IEP apoyada en TIC en el Dpto. del Magdalena. Ciclón.

2 Docente de Ciencias Naturales de la IED Bienvenido Rodríguez. Sede Educativa Col Dptal Bienvenido Rodríguez. Líder del grupo de investigación "Pequeños Investigadores".

3 Docentes pertenecientes al grupo de investigación "Pequeños investigadores" de la IED Bienvenido Rodríguez. Ciclón Magdalena, estrategia $\mathrm{N}^{\circ} 2$. Universidad de la Costa CUC.

- The author; licensee Universidad de la Costa - CUC. Cultura, Educación y Sociedad vol. 9 no. 3, pp. 383-390. Diciembre, 2018 Barranquilla. ISSN 2389-7724 Online 


\section{Introducción}

Esta investigación busca construir saberes y conocimientos a través de la protección y conservación del medio ambiente, fomentando la educación ambiental con el uso adecuado de las relaciones con la naturaleza y de esta manera lograr el bienestar humano a través de prácticas que hagan posible una vida armónica con el entorno en el cual nos desenvolvemos a diario y el cual heredaran nuestros hijos.

Esta investigación inició con la observación de nuestro entorno, vemos con preocupación cómo los desechos son arrojados en lugares no adecuados generando un impacto ambiental negativo que llega hasta las zonas aledañas, se considera que se puede generar una conciencia de selección de esos desechos y convertirlos en productos comercializables.

La visión de esta investigación es innovar, convertir a los investigadores en personas emprendedoras, capaces de desarrollar cualidades y habilidades que les permitan vivir armónicamente con su entorno sin alterarlo en lo posible, tratando siempre de minimizar el impacto de sus acciones sobre él, a la vez que obtiene ganancias de los productos que pueda elaborar con los elementos que son desechados por la comunidad en general. Elementos que al ser expuestos al medio ambiente o arrojado a las fuentes hídricas que posee nuestro municipio generan daños irreparables en los ecosistemas existentes alterándolos irremediablemente, en su mayoría minimizando sus opciones de sobrevivir.

El objetivo general de la presente investigación es identificar estrategias de aprendizaje para promover el cuidado medio ambiental $5^{\circ} 6^{\circ}$ y $9^{\circ}$ IED Bienvenido Rodríguez.

En el municipio de Guamal, situado al sur del departamento del Magdalena se generan grandes volúmenes de desechos sólidos debido a su cantidad de habitantes y al auge económico que posee, también hay elementos del medio al que no se les da un uso adecuado como totumos, cortezas, semillas, se arrojan basuras en sitios no apropiados que no cumplen con los requisitos sanitarios vigentes, otros son arrojados en cinco vías de acceso al municipio, otros desechos son arrojados al rio o caños, otros son quemados generando un serio problema, la comunidad carece de la cultura de reciclaje, no existen políticas ambientales que permitan incentivarlas, ni campañas de sensibilización al respecto, los desechos no tienen un tratamiento que permita minimizar su impacto en el entorno.

En la institución educativa "Bienvenido Rodríguez", aún hay estudiantes que consumen sus alimentos y arrojan papeles, bolsas, vidrios y otros desechos en el suelo, jardines y otros espacios, todo esto nos ha hecho reflexionar y darnos cuenta que muchas veces causamos daño en nuestro entorno sin proponérnoslo, ya sea en el colegio, casa o trabajo, por esta razón decidimos hacer parte de la El cuerpo de maestros de la institución educativa departamental Roberto robles de algarrobal desde la reflexión de la práctica pedagógica.

\section{Educación ambiental}

En la actualidad los problemas medioambientales a los que se enfrenta la humanidad se relacionan con fenómenos como: cambios climáticos, deslizamientos de tierra, terremotos, la emisión de $\mathrm{CO} 2$ a la atmósfera, la erosión, la desertización, la contaminación del agua, la disminución de los recursos naturales y energéticos, la acumulación de residuos sólidos y la explosión demográfica (Schwartz S. 1977).

Esta situación no sólo compete a especialistas en asuntos tecnológicos, biológicos y científicos; también es la consecuen- 
cia de los comportamientos de las personas, quienes en su mayoría no se sienten parte de la situación e identifican las acciones que pueden emprender como individuos para lograr cambios, por el contrario, mediante sus prácticas, provocan un incremento en la gravedad de un problema ambiental; y, por ende, sobre ellas mismas (Herrera, 2014, citando a Martínez-Soto, 2006).

Según Rengifo, Quitiaquez y Mora. (s.f). Hoy en día en nuestro país se ve la necesidad de una educación ambiental que persista en los conocimientos, actitudes, comportamientos y hábitos que nos oriente a conseguir que la población cambie su clásica percepción de que la naturaleza es un elemento pasivo y complaciente, que se regenera automáticamente, porque es un bien del cual vamos a tener por el resto de nuestras vida, siempre disponible para satisfacer las necesidades del ser humano (Herrera y Bravo, 2013).

Esta variación debe producirse mediante un concepto que considere a la naturaleza como un elemento activo, que responde y reacciona ante los estímulos de los seres vivos. Por consiguiente, la estrategia en mención debe promover en la sociedad el aprendizaje y análisis de lo que la naturaleza transmite, además, distinguir que el medio ambiente no en todos los casos puede regenerarse y que las utilidades de los recursos en algún momento ya no son ilimitadas (Touguinas y Pato, 2011).

En nuestro país, se vienen desarrollando acciones junto con el Ministerio de Educación para mejorar el proceso de formación de las presentes y futuras generaciones con la contratación de expertos, realización de diplomados, cursos ,talleres dirigidos a perfeccionar a los docentes en el área de ciencias naturales y la matemática, que ha evidenciado recursos significativos y que han sido evidenciados, adicionalmente, que a la fecha no han arrojado resultados positivos verificables, por otro lado, la Academia de Ciencias de la República Dominicana mostró unas investigación relacionada con "Percepción de la ciencia y la tecnología en estudiantes de bachillerato" donde se deja ver las vivencias que tienen los adolescentes sobre los avances científicos y tecnológicos.

La formación es objeto y espacio donde el sujeto se piensa, se define, se reconoce bajo un componente cultural propio, miembro de una familia particular y en un medio social específico. Es allí donde cada individuo debe encontrar su realización como un ser en armonía. No existe formación, sino porque hay un individuo educable que le da sentido y además el método educativo se debe preparar para esto, su misión es prepararlos para el mundo.

Sobrevivimos por las células muertas, así como una sociedad vive de la muerte de sus individuos, lo que le permite rejuvenecer (Pino, 2013). Las poblaciones han sido participe de cambios que han resultado ser complejos, a los cuales no se estaba del todo preparado, esto, ha traído consigo afectaciones directas en el trabajo, las relaciones y las acciones.

Unas de las particularidades de la sociedad en la que vivimos, tiene que ver con el conocimiento de los temas ambientales las cuales se potencializan en la edad escolar, en un medio de realización humana más que en un fin como ciencia que se debe ser aprendida, y debe brindarle al individuo la posibilidad de superar retos y obstáculos, de proponerse alternativas de solución, de analizar y obrar por un cambio (Gonzales, 2010).

De no ser así se verá obligado a ser conducido y que otros decidan por él. Pero esa realización no es solamente axiológica; "es un desarrollo humano que tienen un fundamento esencial en el desarrollo lógico, definitivo como estrategia para conocer el mundo, apropiar y cambiar su cultura, abordar una situación nueva con la posibilidad de desglosarla (análisis), organizar una información de acuerdo con unas 
prioridades (clasificación y seriación), proponer alternativas de acción (transferencia y síntesis), tomar decisiones y así lograr la autonomía intelectual, fundamento de la autonomía moral“ (Cifuentes, 2013).

Arboleda (2011), argumenta que se debe contemplar tres aspectos importantes en el proceso educativo: a la apropiación social del conocimiento, Fortalecimiento de la comunidad científica, Generación de nuevo conocimiento. En donde se concluye lo importante de lograr que el estudiante aprenda a encontrar nuevas alternativas que conlleve al éxito de las tareas. Por lo tanto, es importante que la ejecución de tareas tenga como fin último el reconocimiento de lo que se debe y no realizar, por lo que es a través de las estrategias didácticas que se fortalece la participación activa, guiando a los estudiantes para que sea creador de lo que desea aprender y se incremente el interés en el área particular.

Por otra parte Núñez (2010), sustenta que parte del gran interés y motivación que se despierta en los niños en el proceso educacional se deriva de su interacción con actividades significativas, donde se observa un mayor desarrollo en cuanto al pensamiento espacial y sistema geométrico, dichas actividades se deben ajustar a la edad e intereses y habilidad de los niños, de igual forma como aspecto muy importante se resalta el interés y habilidad que muestran los niños en lo relacionado con las actividades en el PC.

La Ley 139-01 que rige el Sistema de Educación Superior, Ciencia y Tecnología en su artículo 6 plantea que: la educación superior, la producción y el acceso al conocimiento científico y a las tecnologías, son derechos de todos los ciudadanos y ciudadanas y, por tanto, el desarrollo de las mismas es un servicio público, inherente a la finalidad social del Estado.

Asimismo, es un deber del personal educador contribuir a la excelencia académica de la educación superior y a su sostenimien- to, esto último en la medida de sus posibilidades económicas el Art. 7 de la referida ley, plantea que: la educación superior tiene por finalidad proporcionar formación científica, profesional, humanística, artística y técnica del más alto nivel. “

Se le suma el objetivo de contribuir a la competitividad económica y al desarrollo humano sostenible; promover la generación, Congreso Iberoamericano de Ciencia, Tecnología, Innovación y Educación; "contribuir a la preservación de la cultura nacional, y desarrollar las actitudes y valores que requiere la formación de personas responsables, con conciencia ética y solidaria, reflexivas, innovadoras, críticas, capaces de mejorar la calidad de vida, consolidar el respeto al medio ambiente, a las instituciones del país y a la vigencia del orden democrático" (p. 23).

Con base a lo anterior, para la Universidad Autónoma de Santo Domingo, UASD y los docentes en sintonía con la ley 139-01, se ha convertido en una meta poder lograr la disminución de las dificultades que los estudiantes traen a la escuela, por lo que se deben contemplar en la puesta en marcha de estrategias los recursos adecuados que respondan no solo a las necesidades específicas, sino que además se logren los propósitos, tal como el aprendizaje de las matemáticas articulados con la ciencia.

\section{Aprendizaje}

El aprendizaje según Hernández (2017), "es un conjunto de conocimientos, habilidades, capacidades que parten de conocimientos previos de tal forma que cuando se enlazan los conocimientos nuevos con los previos se encuentra significado a lo que se aprende"(p.p 12); este parte de los intereses personales y también se fundamenta en generar actividades de experiencia vividas en edades tempranas como un cimiento que permita un mejor y adecuado desarrollo cognitivo. 
"Cabe resaltar que el aprendizaje guarda una estrecha relación con el entorno, nunca se encuentra terminado ya que cada vez se aprenden nuevas formas de aprender para comprender la realidad cotidiana que a su vez nunca es estática” (Hernández, 2017).

Ausubel citado en Camacho y Aladro (2011), "establece que los resultados de aprendizaje se clasifican en: la forma en que se le presenta al estudiante los materiales informativos, así como de la forma en la cual el estudiante enlaza o incorpora la información obtenida a su estructura cognitiva; en base a ello se establecen la existencia de diversos tipos de aprendizajes como: Aprendizaje por recepción, por descubrimiento, por repetición o memorístico y el significativo".

La Educación Instrucción según Sarramona (1989) el término educación es de uso común en la vida cotidiana porque a todos afecta de algún modo, existen diversas formas de concebirla, y más aún de materializarla, todas tienen como denominador común la idea de perfeccionamiento, relacionada a un enfoque ideal del hombre y la sociedad.

La educación, aparece como mediadora de los ideales humanos. Se pueden aplicar tres significaciones generales a la educación [11] Como una institución social: El sistema educativo. Es así como se habla de diferentes sistemas de educativos según el contexto histórico-comparativo o el contexto socio-político".

\section{Estilo de Aprendizaje}

Se puede decir que cualquier persona puede aprender dependiendo de la forma o manera en lo cual lo hace; llamándose simplemente estilo de aprendizaje, que:

"Son los rasgos cognitivos, afectivos y fisiológicos que sirven como indicadores relativamente estables, de cómo los alumnos perciben interacciones y responden a sus ambientes de aprendizaje. Las distintas maneras en que unindividuo puede aprender.
Se cree que una mayoría de personas emplea un método particular de interacción, aceptación y procesado de estímulos e información" (EcuRed, s.f).

Para encontrarle significado a las asignaturas necesariamente se debe interpreta y tener en cuenta los ambientes donde se produce el saber, en esta participan las vivencias desde el orden cultural, social, físico, psicológico entre otras. Estos aprendizajes se pueden brindar aulas de clases, laboratorios en donde el estudiante pueda descubrir y transformar lo que le enseñan.

\section{La experimentación en las aulas}

En el trabajo realizado por Bopegedera (2011), fue un logro que permitió mostrar diversas maneras de trabajar desde la experimentación, especialmente en el área de la química, reconociendo la importancia al inicio de los años escolares.

Las actividades realizadas evidencian la mejora del rendimiento académico, logrando el contraste de resultados que reafirman y potencian las estrategias utilizadas. y permite hacer comparaciones con datos estadísticos de este resultado.

Aguilar (2011), coloca en plenaria que él, aprendizaje específicamente de química los laboratorios resultan un factor clave en el ejercicio práctico.

En la publicación de González \& Urzúa (2012), afirman que "el uso de materiales cotidianos, de fácil acceso y bajo costo se puede favorecer la implementación de actividades experimentales en la enseñanza de la Química, disminuyendo así algunos de los factores que las limitan como son: cantidad de reactivos utilizados y los residuos que se generan" p.p 6.

Según Rivera, (2016) es la experimentación una estrategia relevante en el área de ciencias naturales, potenciando habilidades en estudiantes tales como el análisis, atención, capacidad de descubrimiento, generación de resultados, observación constante etc. 
Como estrategia pedagógica entonces, es una nueva oportunidad, creativa y de participación constante siguiendo los lineamientos institucionales, yendo en coherencia y concordancia con los propósitos de la asignatura y los logros esperados.

Por lo tanto, una de las maneras mas oportunas de acercarse al conocimiento a través del método científico motiva a los estudiantes a seguir aprendiendo, si nos desligamos de las cuatro paredes del aula de clases, realizando trabajos con mayor rigor, disciplina y dedicación.

Todo lo antes planteado lleva hacer un seguimiento de la forma en la que los estudiantes articulan competencias del saber y del ser, de acuerdo a los valores inculcados y la manera en la que se relacionan con los compañero,s en la ejecución de tareas al momento de trabajar en equipo, la equidad al momento de repartirse los materiales y la responsabilidad en el cumplimiento de las tareas, podríamos enunciar todo esto en un solo concepto; el interés. (Rivera, 2016).

En síntesis, para entender la labor educativa, es necesario tener en consideración en el proceso educativo. Algunas tendencias del proceso de enseñanza y aprendizaje. Así mismo es esencial propender la participación de los estudiantes en la puesta en marcha de tareas que van más allá de un aprendizaje para una calificación, sino que por el contrario se potencie la postura crítica, el dialogo desde punto de vistas distinto y se articulen resultados interesantes (Ministerio de Educación, Cultura y deporte.2001).

\section{Metodología}

El presente estudio se desarrolla bajo el paradigma empírico analítico, El tipo de investigación Cuantitativa, el diseño es descriptivo de campo no experimental, el tiempo es longitudinal. Ante, el cual, según Ramírez, (2011) Las investigaciones basadas en este paradigma se orientan a la comprobación de hipótesis, considerando la vía hipotética deductiva como la más efectiva para todas las investigaciones.

\section{Población y muestra}

La población participante fueron los estudiantes de los grados $5^{\circ}, 6^{\circ} \mathrm{Y} 9^{\circ}$ la Institución Educativa Bienvenido Rodríguez, con una muestra de 30 estudiantes.

\section{Técnica e instrumentos de recolección de la información}

Para la recolección de la información se aplicó un cuestionario de preguntas cerradas y también se empleó como técnica la observación registrada en un diario de campo.

\section{Procedimiento}

Fase (1): Identificación de la problemática a estudiar.

Fase (2): Definición de la población y muestra.

Fase (3): Definición de las variables de estudio.

Fase (4): Fundamentación teórica.

Fase (5): Escogencia de las técnicas e instrumentos de recolección de información.

Fase (6): Aplicación de los instrumentos.

Fase (7): Análisis y procesamiento de los datos.

Fase (8): Generación de resultados.

Fase (9): Socialización y discusión de los hallazgos encontrados.

\section{Resultados}

Con base a la gráfica anterior se puede inferir que los estudiantes responden lo siguiente:

En alusión a la primera pregunta; ¿En tu colegio se promueven actividades fuera del aula de clases? $\mathrm{El}$ 50\% de los encuestados afirma que si, el 30\% afirma que algunas veces y finalmente el $20 \%$ dice que no.

Con relación a la segunda pregunta: ¿Has participado en experimentos para pro- 
mover el cuidado del medio ambiente? EL $30 \%$ afirma que si, seguido de un $10 \%$ que dice que algunas veces, finalmente un $60 \%$ expone que no ha participado.

La pregunta numero 3: ¿Tus maestros propician espacios novedosos de aprendizaje? El 30\% afirma que si, un 10\% afirma que algunas veces $\mathrm{y}$, finalmente un $60 \%$ afirma que no se propician espacios novedosos de aprendizaje.

Finalmente, en la cuarta pregunta: ¿Se maneja en tu colegio actividades para el cuidado del medio ambiente?, el $20 \%$ afirma que si, el $40 \%$ afirma que algunas veces $y$, finalmente el $40 \%$ afirma que no se manejan actividades para el cuidado del medio ambiente.

\section{Conclusiones}

De acuerdo con los resultados, se puede inferir que, para los procesos de aprendizaje desde cualquier área, especialmente en lo que tiene que ver con el medio ambiente; resulta necesario, novedoso y pertinente los experimentos, contribuyendo al quehacer practico y desde las miradas pedagógicas, favoreciendo el desarrollo del pensamiento crítico en los estudiantes.

De esta manera las prácticas de laboratorio se convierten en una estrategia didáctica que promueve el acercamiento de los estudiantes a las ciencias naturales y favorece el aprendizaje significativo de sus teorías y conceptos.

Es notorio observar que, en la Institución Educativa Bienvenido Rodríguez, los estudiantes dicen que, si bien es cierto que las metodologías de los docentes han cambiado, que hay más interacción en otros escenarios, dejando de lado solo el aula de clases, falta aún la promoción e intensificación de actividades referente a los temas medioambientales, así mismo, se debe incentivar actividades experimentales, analizando conceptos y teorías, y logrando generar resultados aplicables.
Para la realización de trabajos experimentales se debe partir de lo que pretendemos con lo que vamos a trabajar, lo que invita a los involucrados no solo a planear la tarea, sino que además tener en cuenta los recursos que requiere para ejecutarla, así mismo, partir de unos supuestos de lo que posiblemente encontraré y si en realidad contribuye al aprendizaje que se espera con los estudiantes.

En definitiva, es importante inferir que en toda actividad práctica los estudiantes adquieren nuevas habilidades y enseñanzas que emplearan en determinados momentos de su vida.

\section{Referencias}

Avendaño, I., Cortés, O., Guerrero, H. (2015). Competencias sociales y tecnologías de la información y la comunicación como factores asociados al desempeño en estudiantes de básica primaria con experiencia de desplazamiento forzado $\mathrm{Di}$ versitas: Perspectivas en Psicología, 11(1). 13-36. Recuperado de http://www. scielo.org.co/pdf/dpp/v11n1/v11n1a02. pdf.

Bruño, G. (s.f). Elementos de Geometría. París: Bouret.

Camargo, L. (2011). El legado de Piaget a la didáctica de la Geometría. Revista Colombiana de Educación, 60. 41-60

Colorear, F. (2016). Figuras GeométricasDibujos para colorear. Recuperado de http://www.cicloescolar.com/2012/11/figuras-geometricas-dibujos-para.html

Godino, J., Batanero, C. y Roa, R. (2002). Medida de magnitudes y su didáctica para maestros. Granada: Universidad de Nueva Granada.

Grajales D. (2013). Estrategias didácticas para fortalecer el pensamiento geométrico en estudiantes de grado sexto. [Tesis de pregrado]. Universidad Católica de Manizales. Colombia. 
Guerrero, H. (2015) El portafolio: una herramienta facilitadora del cambio en la educación superior desde la práctica didáctica. Revista del Instituto de Estudios en Educación Universidad del Norte, 22. 143-155. http://dx.doi. org/10.14482/zp.22.6340

Guerrero, A. (2006). Desarrollo axiomático. Bogotá, D.C.: Ecoe. Recuperado de https://www.linio.com.co/p/geometri-adesarrollo-axioma-tico-ana-bereniceguerrero-g-vdbfmi

ICFES. (2011). Orientaciones para el examen de estado de la educación media. Recuperado de http://www.slideshare. net/cikey/guia-saber-

Luengo, R. (2005). Proporcionalidad Geométrica y Semejanza. Síntesis, 36 (14).47-71.

Marcos, G. (2008). Un modelo de competencias matemáticas en un entorno interactivo. [Tesis Doctoral]. Universidad de la Rioja, La Rioja.

Moisés, E. (1968). Elementos de Geometría. México, D.F.: UDEA. Recuperado de http://www.biblio.unlp.edu.ar/catalogo/ opac/cgi-bin/pgopac.cgi?VDOC=1.28716

OCDE. (2006). Marco de la evaluación. Conocimientos y habilidades en ciencias, matemáticas y lectura. Recuperado de https://www.oecd.org/pisa/39732471.pdf

Pellegrini, N. y Reyes, R. (2009). Reciclaje de papel en la Universidad Simón Bolívar. Revista de Investigación. 67(33).
República de Colombia. Ministerio de Educación Nacional (2006). Estándares Básicos de Competencias en Lenguaje, Matemáticas, Ciencias y Ciudadanas. Recuperado de https://www.mineducacion.gov.co/1621/articles-340021_recurso_1.pdf

República de Colombia. Ministerio de Educación Nacional (2011). Lineamientos Curriculares. Recuperado de:http:// www.mineducacion.gov.co/lineamientos/matematicas/matematicas.pdf

República de Colombia. Ministerio de Educación, Cultura y Deporte. (2011). La experimentación en la enseñanza de las ciencias. Recuperado de https://sede. educacion.gob.es/publiventa/ PdfServlet?pdf $=$ VP10357.pdf\&area $=\mathrm{E}$

Rivas, L. y Hernández, L. (2010). La transdisciplinariedad en un proyecto de reciclaje. Revista CENIC, 41(4). Recuperado de http://revista.cnic.edu. cu/revistaCQ/articulos/la-transdisciplinariedad-en-un-proyecto-de-reciclaje

Rivera, A. (2016). La experimentación como estrategia para la enseñanza aprendizaje del concepto de materia y sus estados. [Tesis de Maestria]. Universidad Nacional, Manizales.

Salamanca, M., Lombana, N. y Holguín, W. (2010). Uso de la robótica educativa como herramienta en los procesos de enseñanza. Ingeniería Investigación y Desarrollo, 10(1). 